\title{
Determining tumor category of ocular surface squamous neoplasia: science or art?
}

\author{
Curtis E. Margo, Norberto Mancera \\ Department of Ophthalmology, and Pathology and Cell Biology, Morsani College of Medicine, University of South Florida, Tampa, Florida, USA \\ Correspondence to: Curtis E. Margo. Departments of Ophthalmology, and Pathology and Cell Biology, Morsani College of Medicine, University of \\ South Florida, Pathology, 13330 USF Laurel Dr., 4th Floor, Tampa, FL 33612, USA. Email: cmargo@health.usf.edu. \\ Comment on: Singh S, Mohamed A, Kaliki S. Ocular surface squamous neoplasia: analysis based on the 8th American Joint Committee on Cancer \\ classification. Int Ophthalmol 2018. [Epub ahead of print].
}

Submitted Nov 30, 2018. Accepted for publication Dec 10, 2018.

doi: $10.21037 / \mathrm{atm} .2018 .12 .15$

View this article at: http://dx.doi.org/10.21037/atm.2018.12.15

Cancer staging is the process of determining the extent of disease. The goals of staging classification are to plan treatment and assess prognosis. Staging classifications are also valuable in evaluating the results of treatment and in improving communications for clinicians and cancer researchers. The classification of anatomic extent of disease usually employs the TNM system, where T (tumor) category represents the extent of primary tumor by size and depth of invasion of adjacent tissues; criteria are tumor type and site-specific. The $\mathrm{N}$ (node) category specifies the presence and extent of regional node involvement. The $M$ (metastasis) category indicates the presence or absence of distant metastasis. For more than half a century, the TNM classification of malignant tumors has been refined and improved based on iterative insights and criticisms. Refinements are made periodically by internationally recognized experts after studying available evidence. Although the TNM system has been shown to be a robust predictor of clinical outcome for most malignancies, refinement is an ongoing process.

Singh and associates have contributed to this legacy by conducting a retrospective study to test the validity of the American Joint Committee on Cancer (AJCC), 8th edition staging of ocular surface squamous neoplasia (OSSN) of conjunctiva (1). Using TNM staging for conjunctival carcinoma, the authors staged 136 cases of OSSN from 127 patients, for which they had a mean follow-up of 15 months. Their findings (and conclusions) were both expected and surprising. As one might anticipate, increasing tumor $(\mathrm{T})$ category was associated with more advanced disease. On the other hand, the majority of cases (78\%) were T3, which corresponds to squamous cell carcinoma (SCC) that invades adjacent structure. All tumor recurrences occurred among T3 patients (19 cases, or 14\%). There were no T1 stage tumors among 136 cases. The authors concluded that the existing AJCC staging can be improved upon and suggested that separate clinical and pathologic classifications may be helpful. We interpret the results of this study as illustrative of a greater dilemma with AJCC staging of conjunctival carcinoma-that of unintentional complexity that can lead to systematic misclassification of $\mathrm{T}$ category.

We need to start with a focused review of definitions of primary tumor " $T$ " categories according the AJCC 8th edition, which is determined by site-specific rules for conjunctival carcinoma (2). Tis is carcinoma in situ, the preinvasive phase of SCC. This stage of conjunctival intraepithelial neoplasia ranges from mild and moderate dysplasia to severe dysplasia and carcinoma in situ. Without access to lymphatics or blood vessels, the intraepithelial stage of squamous neoplasia is biologically a pre-cancer, incapable of regional or distant metastasis because it cannot spread distantly. Once the in situ squamous neoplasia breaches the basement membrane, it becomes SCC. Category T1 is SCC equal or less than $5 \mathrm{~mm}$ in diameter without invasion of adjacent structures, and T2 


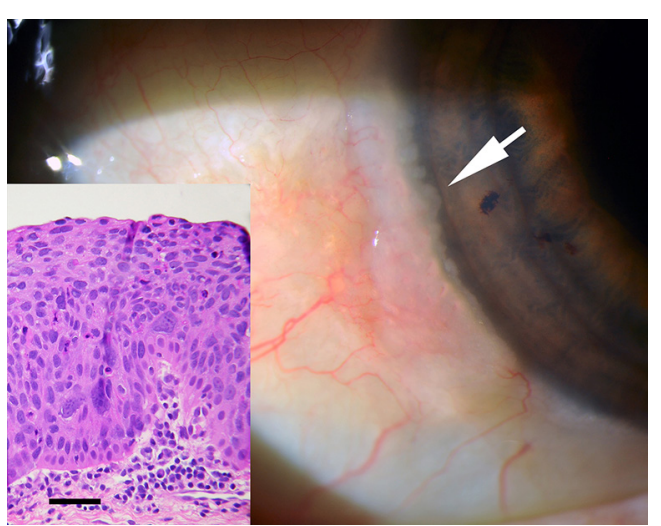

Figure $1 \mathrm{~A}$ buff colored mass is present at the limbus from 7 to 9 o'clock. It is free movable over sclera with conjunctiva normally tethered at the limbus. Contiguous peripheral corneal has a frosted glass appearance with scalloped edges (arrows). The excisional biopsy showed carcinoma in situ (inset) (hematoxylin-esoin; bar $=110 \mu \mathrm{m})$. The lesion receives a Tis category classification.

is SCC greater than $5 \mathrm{~mm}$ without invasion of adjacent structures. Adjacent structures include cornea, intraocular compartment, forniceal, palpebral, tarsal conjunctiva, lacrimal punctum, canaliculi, plica, caruncle, anterior or posterior eyelid lamella, and eyelid margin. Category T3 is SCC with invasion of any of the aforementioned adjacent structures but short of the orbit. T4 is defined by orbital invasion without further extension. This category is also subdivided but these divisions will not be discussed.

With that background, Singh and associates placed 113 cases of OSSN in T3 and recorded the histopathologic features of 94 cases in Table 5. Of those 94 cases, 31 (33\%) were SCC, and the rest were carcinoma in situ or squamous epithelial dysplasia. According to the definition of category T3, however, all cases must be SCC. Why the discrepancy? Singh and associates explain their rational in the Discussion section: the "Majority of OSSN bave their epicenter at limbus, which results in their categorization under T3 category despite being carcinoma in situ and smaller size." (1). We disagree with this interpretation. In situ neoplasia should not be categorized as T3 just because it is located at the limbus or any other anatomic location for that matter. However, we also realize that TNM criteria for OSSN have been subject to differing interpretations.

Systematic mis-categorization of in situ conjunctival intraepithelial neoplasia has plagued some of the largest clinical studies found in the literature (3). The reasons for misclassification may vary but often involve assigning in situ carcinoma and squamous epithelial dysplasia to an invasive (i.e., SCC) T category. The implications of systematic miscoding in this direction are substantial. Take, for example, a matched case-control study designed to compare the effectiveness of primary surgical versus medical treatment of OSSN (4). The study involved 98 patients that were equally divided into surgical and medical treatment groups. All 98 patients were determined to have $\mathrm{T}$ categories that fell between $\mathrm{T} 1$ and $\mathrm{T} 3$. That means all cases were SCC of conjunctiva. Pathologic assessment of the 66 patients that had biopsies, however, showed only 3 SCCs. The remainder ranged from mild dysplasia to carcinoma in situ. Anyone that casually reads the paper would understand the conclusion as medical therapy for conjunctival SCC is equally effective as surgical excision. This inference is incorrect. Only 3 cases of 98 were SCC (invasive disease) and they were all treated surgically. Therefore, no conclusion regarding the effectiveness of medical treatment for SCC can be drawn from this study.

If one examines the latest revisions in AJCC 8th edition on conjunctival carcinoma, it includes the new stipulation that $\mathrm{T} 1$ and $\mathrm{T} 2$ include invasion of the conjunctival basement membrane (2). This provision is a redundancy since all conjunctival carcinomas $\mathrm{T} 1$ and above must breach the basement membrane if they are diagnosed SCC. The proviso was likely added to emphasize that by definition $\mathrm{T} 1$ and T2 must represent invasive disease, or SCC. The same holds for T3.

Singh and colleagues correctly point out that Tis, T1 and $\mathrm{T} 2$ cannot be easily distinguished from one another on clinical examination alone. Their difficulty, however, involved interpreting OSSN at the limbus, where a majority of OSSN originate. They classified limbal lesions as T3 even when histologically diagnosed as intraepithelial dysplasia or carcinoma in situ. Why might this happen?

Let's examine a case to get a better idea of the relevant anatomy. Figure 1 shows the clinical appearance of a suspicious lesion extending around the limbus from 7 to 9 o'clock. The slightly elevated mass with a patchy buff color, ill-defined borders, and mild vascularization was freely moveable over sclera. The lesion extended on to cornea where it had a frosted glass appearance with 


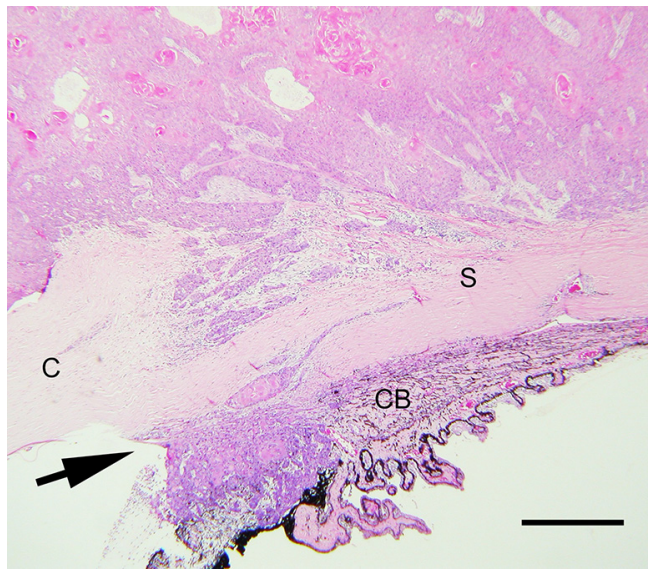

Figure 2 A neglected, well-differentiated squamous cell carcinoma of the limbus. The tumor sits atop of cornea (C) and sclera (S), and invades the eye. Tumor is present in the ciliary body (CB) and is visible in the angle (arrow). The lesion invaded anterior orbit and was designated T4a (hematoxylin-eosin; bar $=200 \mu \mathrm{m}$ ).

scalloped margins. The clinician excised the lesion with 1-2 mm of presumed uninvolved margins on the conjunctival side. Histologically the lesion was carcinoma in situ (Figure 1, inset); the conjunctival surgical margin was free of tumor. The limbal margin, however, was a different matter. Knowing that abnormal cornea epithelium remained after the limbal margin was cut through, the surgeon scraped the frosted epithelium from Bowman's layer then applied absolute alcohol to the area. Bowman's layer appeared normal. Some surgeons submit the chafed epithelium in formalin for microscopic confirmation of dysplasia or carcinoma in situ; others do not (corneal epithelium was not submitted in this case). The final diagnosis was intraepithelial squamous neoplasia, carcinoma in situ. It is category Tis.

A basement membrane lies beneath both conjunctival and corneal epithelium, but corneal basement membrane is usually multilaminar while conjunctiva is thinner and more discrete. The conjunctival dissection of suspected carcinoma in situ cannot be continued at the limbus because fascia bulbi (capsule of Tenon) is anchored to sclera. Short of performing a lamellar scleral-corneal dissection, the limbal surgical margin of most conjunctival intraepithelial squamous neoplasms will appear involved. The important point, however, is that if the conjunctival margin contains intraepithelial squamous neoplasia, the cornea can be assumed free of invasive disease if corneal debridement results in a smooth, clear Bowman's layer.

This is not to say that T3 or higher stages of SCC cannot arise at the limbus. Even well-differentiated SCCs can invade adjacent tissues from this location as illustrated in Figure 2.

TNM classifications serve a worldwide audience of professionals involved in the care of cancer patients. The process of staging needs to be intuitive yet sophisticated enough to collect data that clinicians and cancer researchers can use to predict outcomes and advance better treatment strategies. Developing the ideal classification and staging process will always be a work in progress.

\section{Acknowledgements}

None.

\section{Footnote}

Conflicts of Interest: The authors have no conflicts of interest to declare.

\section{References}

1. Singh S, Mohamed A, Kaliki S. Ocular surface squamous neoplasia: analysis based on the 8th American Joint Committee on Cancer classification. Int Ophthalmol 2018. [Epub ahead of print].

2. Conway RM, Graue GF, Pelayes D, et al. Conjunctival carcinoma. In: Amin MB, Edge SB, Greene FL, et al. editors. AJCC Cancer staging manual. 8th edition. Switzerland: Springer, 2017:787-93.

3. Margo CE, White AA. Ocular surface squamous neoplasia: terminology that is conceptually friendly but clinically perilous. Eye (Lond) 2014;28:507-9.

4. Nanji AA, Moon CS, Galor A, et al. Surgical versus medical treatment of ocular surface squamous neoplasia: a comparison of recurrences and complications. Ophthalmology 2014;121:994-1000.

Cite this article as: Margo CE, Mancera N. Determining tumor category of ocular surface squamous neoplasia: science or art? Ann Transl Med 2018;6(Suppl 2):S121. doi: 10.21037/ atm.2018.12.15 